INTERMEDIATE POLARS 


\title{
THE INTERMEDIATE POLARS
}

\author{
COEL HELLIER \\ Department of Physics, Keele University, Keele, \\ Staffordshire, ST5 5BG, UK
}

\begin{abstract}
Recently discovered intermediate polars extend the limits of known behaviour. They now fill the range between, and overlap with, nonmagnetic CVs and AM Hers. They have a wide range of asynchronism, a full range of field strengths, and several different accretion modes and pulsation mechanisms. I highlight (i) a puzzle over the symmetry between magnetic poles, (ii) the X-ray spectra, (iii) the similarity with polars, and (iv) the mass of EX Hya.
\end{abstract}

\section{The known intermediate polars}

Patterson (1994) provided a comprehensive review of intermediate polars (IPs or DQ Her stars), while my Capetown review (Hellier 1995) focussed on the accretion geometry. Here I do not try to be comprehensive, but instead highlight issues which need updating or further attention.

The significant change since Patterson's parade of the 'cast list' has been the addition of new IPs discovered by ROSAT. In addition to the WFC source PQ Gem (RE0751+14; Mason et al. 1992) we have the XRT sources RX 0558+53 (Haberl et al. 1994), RX 1712-24 (Buckley et al. 1995), RX $1238-38$ (Buckley 1995) and probably RX 0028+59, RX 0153+74 and RX 1914+24 (Haberl \& Motch 1995), with further identifications likely.

With these additions I present the known IPs in Table 1. The list is conservative, including only certain or almost certain members, placing most weight on a convincing $\mathrm{X}$-ray periodicity, since this is the hardest IP signature to fake.

It has become traditional to plot IPs on a spin-orbit diagram (Fig. 1) and then draw conclusions from it (e.g. King \& Lasota 1991 and references therein). The previously touted $\mathrm{P}_{\text {spin }}=0.1 \mathrm{P}_{\text {orb }}$ relation is not apparent in up-to-date IP lists - indeed least-square fits produce a roughly orthogonal line. The safest interpretation is as a scatter diagram with three outliers. 
TABLE 1. The known intermediate polars.

\begin{tabular}{lcccc}
\hline Star & $\mathrm{P}_{\text {spin }}(\mathrm{s})$ & $\mathrm{P}_{\text {orb }}(\mathrm{h})$ & $\mathrm{M}_{\mathrm{V}}$ & Mag Field (MG) \\
\hline EX Hya & 4022 & 1.638 & 13 & $\sim 1$ \\
RX 1238-38 & $1850:$ & $3.7:$ & & \\
TX Col & 1911 & 5.72 & 15 & $"$ \\
TV Col & 1910 & 5.49 & 14 & $"$ \\
1E 0830.9-2238 & 1558 & & 17 & \\
RX 0153+74 & 1414 & & & \\
FO Aqr & 1254 & 4.85 & 13.5 & $"$ \\
RX 1712-24 & 927 & $3.4:$ & 14 & 8 \\
BG CMi & 913 & 3.23 & 14.5 & 4 \\
PQ Gem & 833 & 5.19 & 14.5 & 12 \\
AO Psc & 805 & 3.59 & 13.5 & $\sim 1$ \\
V1223 Sgr & 746 & 3.37 & 13 & $"$ \\
RX 1914+24 & 568 & & $>19$ & \\
RX 0558+53 & 545 & 4.15 & 14.6 & $"$ \\
YY Dra & 529 & 3.96 & 11 & $"$ \\
GK Per & 351 & 47.9 & 14 & $"$ \\
RX 0028+59 & 313 & $5:$ & 14.6 & \\
XY Ari & 206 & 6.06 & $>23$ & $"$ \\
DQ Her & 142 & 4.65 & 15 & 0.14 \\
AE Aqr & 33 & 9.88 & 11 & 0.3 \\
\hline
\end{tabular}

Notes: References in Sect. 1, with Allan et al. $1996(\mathrm{RX} 0558+53)$ and O'Donoghue et al. 1996 (1E 0830.9-2238). Following Zhang et al. (1995) we quote DQ Her's period as $142 \mathrm{~s}$, however $71 \mathrm{~s}$ is also possible. Magnetic field estimates are reliable only where direct evidence of the field is seen (BG CMi, PQ Gem, RX1712-24). Values are from Piirola et al. 1993 (PQ Gem); Buckley et al. 1995 (RX 1712-24); Chanmugam et al. 1990 (BG CMi); Zhang et al. 1995 (DQ Her). Other systems are likely to have magnetic fields of $\sim 1 \mathrm{MG}$ (see Patterson 1994 for further details).

However, interpreting the $\mathrm{P}_{\text {spin }}=0.1 \mathrm{P}_{\text {orb }}$ line as an upper limit for disc-fed accretors is also tenable: the only systems certainly in the zone between this line and the AM Her synchronism line are EX Hya, which is spinning up rapidly towards the other IPs, and the asynchronous polars such 


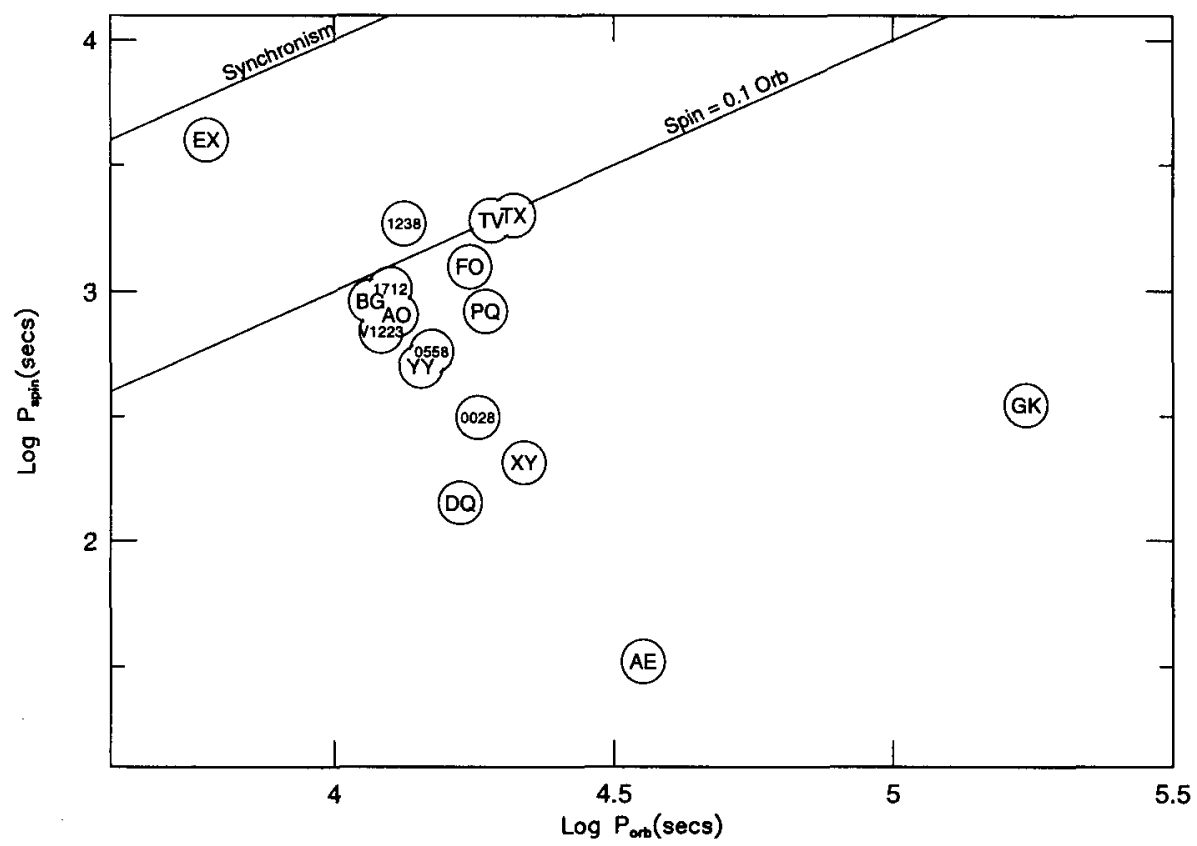

Figure 1. An update of the spin-orbit diagram from Patterson (1994).

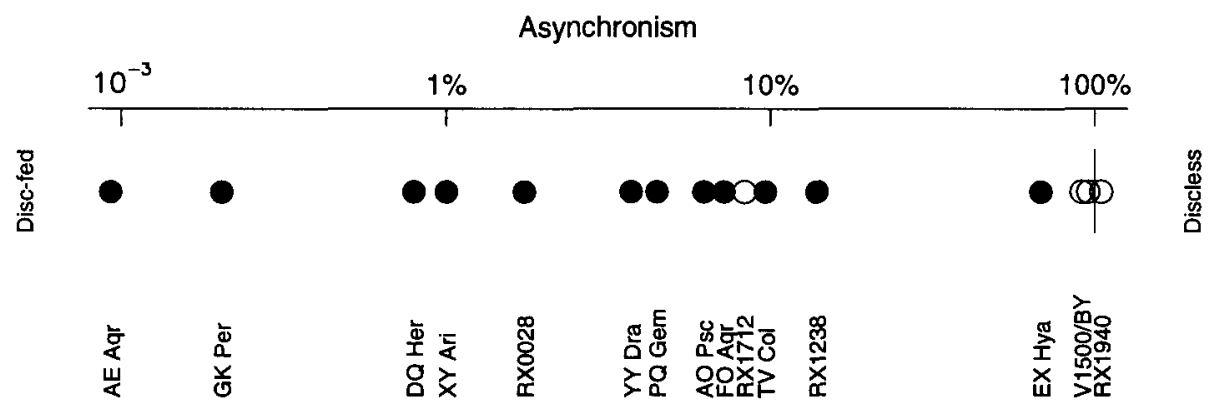

Figure 2. The degree of asynchronism in magnetic CVs. Disc-fed systems are filled symbols, discless systems are open symbols.

as V1500 Cyg, which is heading rapidly for synchronism. In contrast the zone below the $\mathrm{P}_{\text {spin }}=0.1 \mathrm{P}_{\text {orb }}$ line is well populated. This pattern fits the model by Wu \& Wickramasinghe (1991) well. The range of synchronism for magnetic CVs is illustrated in Fig. 2. 


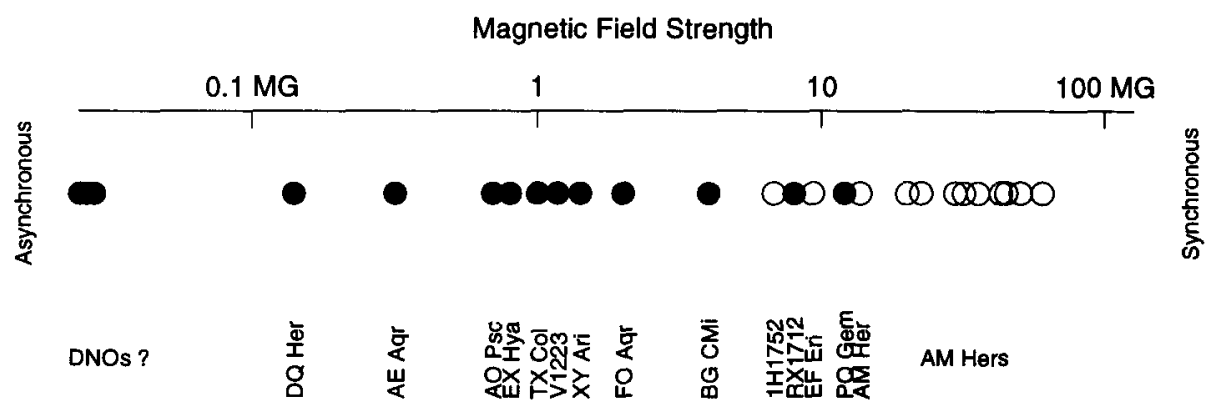

Figure 3. Field strength of magnetic cataclysmic variables (open circles are AM Hers).

\section{A disparate bunch}

The newly discovered systems make it clearer that IPs are not a homogeneous set, but have properties ranging from the non-magnetic CVs to the AM Hers, and even overlapping to some extent. Thus a 'typical IP' model will not apply to many members. Further, magnetic CVs can change modes over time, and, as emphasized by Wynn, King \& Horne (1995), history can be as important as basic characteristics such as field strength in determining the current appearance of a system.

\subsection{RANGE OF FIELD STRENGTHS}

In addition to the range of synchronism, over 3 orders of magnitude, there is a great range of field strengths. BG CMi's status as the most polarized IP has been superseded by PQ Gem and RX 1712-24, both showing polarisation variations over the spin cycle. The implied fields are $\sim 10 \mathrm{MG}$, overlapping with the lower end of the AM Her field distribution (Fig. 3). DQ Her and AE Aqr probably have fields two orders of magnitude weaker. As discussed by Warner (1995), the dwarf nova oscillations indicate a field strength another order of magnitude lower still in 'non-magnetic' CVs. V533 Her could be the 'overlap' between magnetic and other CVs.

\subsection{RANGE OF ACCRETION MODES}

IPs have traditionally been thought to have accretion discs truncated by the magnetic field in the inner regions. While this probably holds for the majority of known IPs, it cannot be the whole story. Buckley et al.'s (1995) discovery of RX 1712-24 presents us with a system showing all the hallmarks of an IP with a spin period of $1003 \mathrm{~s}$ - except for polarization 
pulsed at $927 \mathrm{~s}$, implying that $927 \mathrm{~s}$ must be the spin period and that $1003 \mathrm{~s}$ is therefore the beat period. Variations at the beat period, caused by the orbitally-locked accretion stream flipping between poles, had been proposed as the signature of discless IPs (e.g. Hellier 1991), suggesting that RX $1712-24$ is the first of the discless IPs predicted by Hameury, King \& Lasota (1986).

FO Aqr shows a dominant X-ray pulse at the spin period, and also a weaker, variable, beat period pulsation (Norton et al. 1992). Thus it might be accreting via a hybrid 'disc-overflow' mode, proposed for IPs by Hellier et al. (1989) and Hellier (1993).

As if three different modes weren't sufficient, there is a fourth possibility. Wynn \& King (1995) have suggested that AE Aqr is an 'ejector' in which most of the mass transfer stream is ejected from the system, and only a minority accreted.

Future work will determine the relative incidence of different accretion modes in IPs. Although current evidence points to a truncated disc as most common (e.g. Hellier 1991), this is not necessarily the final word. Notice that if the field in $\mathrm{RX} 1712-24$ were lower, so that no polarization were observed, we would have no inkling of the 927-s period, and might suppose that it was a normal IP with a spin period of $1003 \mathrm{~s}$. Thus other systems could be similarly mis-identified. Careful comparison of RX 1712-24 with other IPs might show whether or not this is the case.

\subsection{RANGE OF PULSE MECHANISMS}

In $\mathrm{AE}$ Aqr the high temperature of the optical/UV spin-pulsation implies that it originates from a region only $\sim 3 \%$ of the white dwarf area, presumably the polecaps. However, in other IPs the evidence instead points towards the 'accretion curtain' model of Hellier et al. (1987), where the pulsations arise in the curtains of material between the inner disc and the white dwarf. Both the temperature of the pulsed emission in FO Aqr and the phase wandering of the optical eclipse in EX Hya are strong evidence for the accretion curtain model in these stars (see Hellier 1995 for further details). DQ Her is different again, with the observed pulsation arising from the entire disc (Patterson, Robinson \& Nather 1978). For a thorough investigation of pulse temperatures see Welsh \& Martell (1996).

The explanation for the differences is likely to be the relative size of the accretion curtains - a slower rotator will have a larger magnetosphere (assuming equilibrium rotation) and so have large curtains, cool at their outer edges. A faster rotator such as AE Aqr would have smaller curtains, so the pulsed flux would be dominated by hotter regions nearer to or on the white dwarf. The same mechanism probably explains the correlation 
between pulse amplitude and spin period noted by Patterson (1994): all IPs have the hot pulse from the inner regions, but in slower rotators the larger curtains add in a large, cooler pulse. This curtain model implies that pulses generally have a range of temperature and can only be characterised with a single temperature in cases such as $\mathrm{AE} \mathrm{Aqr}$, where the pulsation comes from one small region.

In DQ Her we are probably not seeing these regions, due to the high inclination, so we only see a much weaker component reflected off the disc.

\section{Single or double humped pulses?}

An issue which has recently come to prominence is whether an IP produces single or doubled peaked pulses every white dwarf rotation. The old ideas in which X-ray/optical maxima occur when a pole points towards us suggest that a system with one dominant pole would produce a roughly sinusoidal profile, while a system with two bright poles would produce a double peaked pulse. In contrast the accretion curtain model (with pulse maximum when the upper pole points away) predicts a sinusoidal pulse from both one and two pole systems, since in this model the poles act in phase.

This is almost certainly the case for EX Hya, where both the partial $\mathrm{X}$-ray eclipse and the width of the optical emission lines insist that we are seeing roughly equal emission from two poles (see e.g. Hellier 1995 for more details) yet the pulsation is single peaked at the $67-\mathrm{m}$ spin period (unless one wants to postulate that the spin period is actually $134 \mathrm{~m}$, far longer than the orbital period).

However, Zhang et al. (1995) have revisited the phase-shift through eclipse of the 71-s pulse in DQ Her. Using a new technique to boost the pulse's signal-to-noise ratio they show convincingly that the disc must be illuminated by two beams with a rotation speed of $142 \mathrm{~s}$ (rather than 1 beam rotating at $71 \mathrm{~s}$ ). However, since there is no direct sign of a 142-s pulse, the two beams must be equally intense to high accuracy. Thus we have the opposite of EX Hya - a spin-pulse with all the power in the first harmonic rather than in the fundamental.

The only likely way two beams could illuminate the disc equally is if the dipole were tilted at $90^{\circ}$ and is spinning in the orbital plane. However, the phenomenon is more widespread than DQ Her: both RX 0558+53 (Allan et al. 1996) and YY Dra (Haswell \& Patterson 1996) show strong power at the first harmonic, although the fundamental is also present in these stars. Thus, further investigation of the pulse shape from an IP is required to understand why this is seen in some systems and not in others.

The basic ideas of the accretion curtain model (that spin maximum occurs when the upper pole points away; that optical and X-ray maxima are 
in phase; that opacity in the curtain and its changing projected area are the cause of the pulse; Hellier et al. 1987; Rosen, Mason \& Cordova 1988; Hellier, Cropper \& Mason 1991) have recently received strong support from the modelling by Kim \& Beuermann $(1995,1996)$. They reproduce all these features, confirming that the two-pole accretion curtain geometry gives rise to a single-peaked pulse, at maximum when the upper pole points away. Thus we now need to explain the double humps in DQ Her, YY Dra and $\mathrm{RX} 0558+53$. One clue is that systems furthest from synchronism are likeliest to show double-peaked pulses. In addition to these three stars, AE Aqr, PQ Gem and XY Ari also show double humps to some degree, and all six are in the same region of Fig. 1. Double humps arise if the optical depth in the accretion polecap is greatest horizontally (poles acting anti-phased) while single pulses arise if the optical depth is greatest vertically (as in the accretion curtain model, with poles acting in phase). Possibly, rapid rotators, having relatively smaller accretion curtains, are more likely to have light curves dominated by the accretion polecap, producing double humps.

\section{The X-ray spectra}

Low resolution data from the previous generation of $\mathrm{X}$-ray satellites showed that IP spectra were $10-\mathbf{3 0} \mathrm{keV}$ bremsstrahlungs with heavy but patchy absorption (e.g. Ishida 1991). Typical spin pulses are sinusoidal, with minima caused by increased absorption (although stars such as PQ Gem and FO Aqr also show other effects).

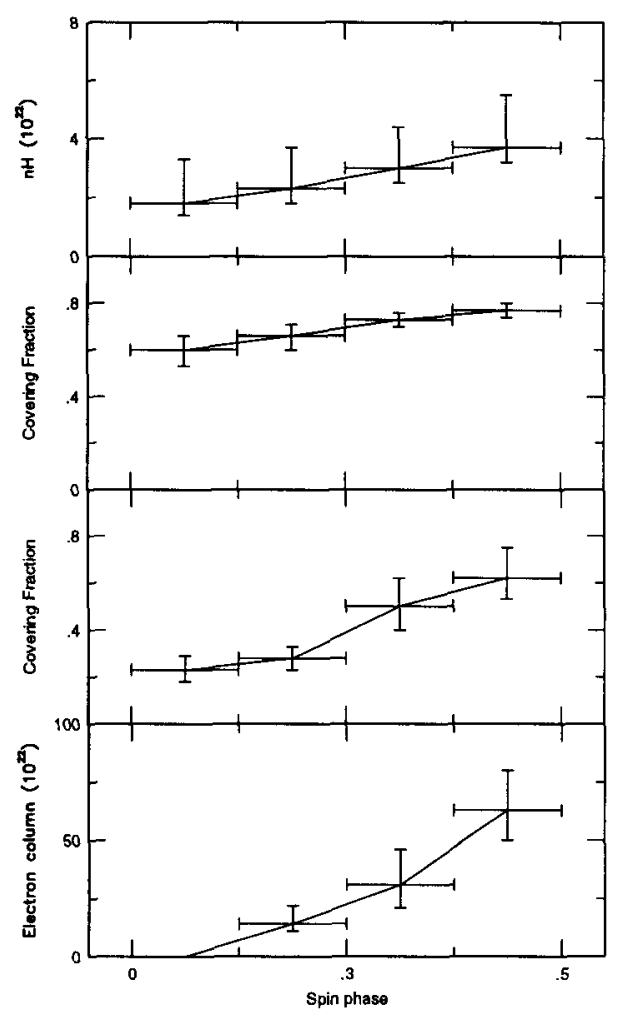

Figure 4: Variation in absorption in AO Psc to reproduce the spin pulse. One absorbing component varies in both density (panel 1; top) and covering fraction (panel 2). A second absorber of density $210^{23} \mathrm{~cm}^{-2}$ varies in covering fraction (panel 3), while a 'grey absorber' varies in density (panel 4). 
So far, ASCA results confirm this picture, their main impact being to increase the complexity of the required absorption. For instance Mukai, Ishida \& Osborne (1994) model the phase-averaged spectrum of FO Aqr with a bremsstrahlung absorbed by three different columns. Hellier et al. (1996) attempted phase-resolved analysis of ASCA data on AO Psc, a star showing a particularly strong and simple, sinusoidal pulse. Given an underlying bremsstrahlung and an interstellar column of $10^{21} \mathrm{~cm}^{-2}$, the pulsation is largely reproduced by a partially covered $\sim 310^{22} \mathrm{~cm}^{-2}$ absorber, increasing in both column and covering fraction during minima (Fig. 4). In addition, though, they require a denser absorber to reduce the high energy flux at minimum. Given the limited energy range of ASCA, the parameterisation is not unique - one possibility combines a $210^{23} \mathrm{~cm}^{-2}$ absorber, with varying covering fraction, and a 'grey' electron scattering column, denser at spin minimum. Such divisions between components are probably artificial; improved data will likely require increasingly complicated distributions of absorbers. Although the need for larger columns and covering fractions during minima is robust, the exact parameterisation is (so far) ambiguous.

The interpretation is still uncertain: it is probable that the accretion columns are tall with a small footprint, producing most absorption along their length, so that flux minimum occurs when the upper pole points towards us. To reproduce the leaky absorbers the material in the flow must be clumpy. However, so far we cannot determine whether the grey-ish absorber is highly ionized material beneath the accretion shock, producing opacity by electron scattering, or dense, opaque blobs obscuring a significant fraction of the lines of sight. Similarly, we don't yet know whether all significant absorption occurs near to the accretion shock, or whether material much further out, where material rises from the plane along field lines, also plays a role. Progress in resolving such issues is likely to come from comparing ASCA data on several systems.

\section{Comparison with AM Hers}

Recent discoveries make IPs look increasingly similar to AM Hers: we now have asynchronous polars and IPs with polar-like field strengths, while the hard X-ray spectrum of both IPs and polars is a bremsstrahlung, showing greater absorption when the pole points towards us. Further, ROSAT has discovered a soft X-ray component in some IPs (Haberl \& Motch 1995). Probably this component is present in all IPs, but at too low an energy to be seen in many systems.

This emission is the counterpart of the soft component ubiquitous in AM Hers, where it is blackbody emission from the white dwarf polecap, 
heated both by reflection of hard X-rays and by thermalising of blobs which bury into the surface. Simple interpretation of the ROSAT IP data yields heated areas only $\sim 10^{-5}$ of the white dwarf surface (e.g. Haberl et al. 1994) - small even by the standards of small polecap models. If this is the size of the accretion footprint then electron scattering in the accretion column must be substantial.

Given these similarities there are two significant differences between polars and IPs. The first is that no IP shows an 'off-phase', as occurs every cycle in AM Hers, if the dominant accreting pole is occulted by the white dwarf. The interpretation has to be that accretion is roughly even between the two poles, with little dependence on orbital phase, so that the pole facing us is always accreting.

Further, the extreme flickering characteristic of polars, caused by individual blobs of material hitting the white dwarf, is not seen in IPs. A possible explanation is that the higher accretion rate in IPs causes more blobs to be present at any one time, effectively smoothing the flickering.

Alternatively, the accretion could be much less blobby in an IP (although some blobbiness is required to explain the partial covering in the $\mathrm{X}$-ray spectra). If accreting material were stripped from a disc, rather than coming from a blobby stream as in polars, it could account for the difference. This might further explain why the soft component is much weaker in IPs (no blobby accretion, therefore no soft excess), and, if smoother accretion were more effectively channelled by the magnetic field, why the estimate of the accretion area is so small. A snag with such an argument is that the discless IP RX 1712-24, which is presumably more AM Her-like, does not, so far, have a detected soft component.

\section{The mass of EX Hya}

On a different topic to finish, the high resolution X-ray spectra from ASCA provide a new way of measuring the mass of a white dwarf. The emission line ratios give the plasma temperature of the accretion shock, which, through accretion shock theory and a mass-radius relationship, yields the white dwarf mass (e.g. Ishida \& Fujimoto 1995). For EX Hya, which has the strongest emission lines and so the most reliable result, this method gives a white dwarf mass of $0.47 \pm 0.04 \mathrm{M}_{\odot}$ (Fujimoto \& Ishida 1995).

EX Hya is also the best IP for measuring the mass by traditional means, being an eclipsing double-line binary. Thus we have $i=77^{\circ} \pm 1^{\circ}, K_{1}=69 \pm$ $9 \mathrm{~km} \mathrm{~s}^{-1}$ (Hellier et al. 1987) and $K_{2}=356 \pm 4 \mathrm{~km} \mathrm{~s}^{-1}$ (Smith, CollierCameron \& Tucknott 1993). Combining these gives a white dwarf mass of $0.49 \pm 0.03 \mathrm{M}_{\odot}$ and a secondary mass of $0.095 \pm 0.013 \mathrm{M}_{\odot}$. The agreement with the ASCA result is remarkable given the tight error bars on both 
determinations.

I make three remarks on the masses. Firstly, the secondary mass is lower than the $0.128 \mathrm{M}_{\odot}$ predicted by Patterson's (1984) period-mass relation. Secondly, the secondary mass is very much lower than suggested by recent infrared work by Dhillon et al. (1996). They find spectral features consistent with an M3 or M4 dwarf, which normally has a mass of $\sim 0.3 \mathrm{M}_{\odot}$. Lastly, the white dwarf mass implies an escape velocity of $3600 \mathrm{~km} \mathrm{~s}^{-1}$. The optical emission line wing extends to almost this velocity (for example Hellier et al. 1987 quoted widths of $\pm 3500 \mathrm{~km} \mathrm{~s}^{-1}$ ). Thus the mass can only be correct if we are seeing emission from infalling material which is (i) extremely close to the white dwarf, and (ii) has not been significantly slowed in its journey.

\section{References}

Allan, A., Horne, K., Hellier, C., et al., 1996, MNRAS, in press

Buckley, D.A.H., 1995, private communication

Buckley, D.A.H., Sekiguchi, K., Motch, C., et al., 1995, MNRAS, 275, 1028

Chanmugam, G., Frank, J., King, A.R., Lasota, J.P., 1990, Ap. J., 350, L13

Dhillon, V.S. et al., 1996, in preparation

Fujimoto, R., Ishida, M., 1995, ASP Conf series, 85, 136

Haberl, F., Thorstensen, J.R., Motch, C., et al., 1994, A\&A, 291, 171

Haberl, F., Motch, C., 1995, A\&A, 297, L37

Hameury, J.-M., King, A.R., Lasota, J.-P., 1986, MNRAS, 218, 695

Haswell, C.A., Patterson, J., 1996, these proceedings, p169

Hellier, C., 1991, MNRAS, 251, 693

Hellier, C., 1993, MNRAS, 265, L35

Hellier, C., 1995, ASP Conf series, 85, 185

Hellier, C., Mason, K.O., Rosen, S.R., Córdova, F.A., 1987, MNRAS, 228, 463

Hellier, C., Mason, K.O., Smale, A.P., et al., 1989, MNRAS, 238, 1107

Hellier, C., Cropper, M.S., Mason, K.O., 1991, MNRAS, 248, 233

Hellier, C., Mukai, K., Ishida, M., Fujimoto, R., 1996, MNRAS, in press

Ishida, M., 1991, Ph.D. thesis, University of Tokyo

Ishida, M., Fujimoto, R., 1995, ASP Conf series, 85, 132

Kim, Y., Beuermann, K., 1995, A\&A, 298, 165

Kim, Y., Beuermann, K., 1996, A\&A, in press

King, A.R., Lasota, J.P., 1991, Ap. J., 378, 674

Mason, K.O., Watson, M.G., Ponman, T.J., et al., 1992, MNRAS, 258, 749

Mukai, K., Ishida, M., Osborne, J.P., 1994, PASJ, 46, L87

Norton, A.J., Watson, M.G., King, A.R., et al., 1992, MNRAS, 254, 705

O'Donoghue D., Koen C., Kilkenny D., 1996, MNRAS, 278, 1075

Patterson, J., 1984, Ap. J. Supp., 54, 443

Patterson, J., 1994, PASP, 106, 209

Patterson, J., Robinson, E.L., Nather, R.E., 1978, Ap. J., 224, 570

Piirola, V., Hakala, P., Coyne, S.J., 1993, Ap. J., 410, L10

Rosen, S.R., Mason, K.O. Córdova, F.A., 1988, MNRAS, 231, 549

Smith, R.C., Collier-Cameron, A., Tucknott, D.S., 1993, in "Cataclysmic Variables and

Related Physics", eds O. Regev, G. Shaviv, Ann. Israel Phys. Soc., 10, 70

Warner, B., 1995, ASP Conf series, 85, 343

Welsh, W.F., Martell, P., 1996, MNRAS, submitted

Wu, K., Wickramasinghe, D.T., 1991, MNRAS, 252, 386

Wynn, G., King, A.R., Horne, K. 1995, ASP Conf series, 85, 196

Zhang, E., Robinson, E.L., Stiening, R.F., Horne, K., 1995, Ap. J., 454, 447 